[3] F. W. J. Bоекноuт et J. J. Ott de Vries. Rev. Gén. Lait, 1907, VI, 313, 345 ; 1909 , VII, 385 ,

[4] W. Van Dam. Rev. Gén. Lait, 1910, VIII, 169; 1911, IX, 1; 1912, IX, 151.

[5] Orda-Jensenn. Dairy Bacteriology. J. et A. Churchill, Londres, 1931, p. 150 et 165 .

[6] G. Koxstuer. Landw., Jahrb. Schweiz., 1932, 46, 51 et 1933, 47, 154.

[7] P. D. Watson. J. Dairy Sci., 1929, XII, 289.

[8] A. Peter et F. BADOUX. Manuel de la fabrication du fromage d'Emmental. Hoirs K. J. Wyss S. A., Berne, 1937, p. 96 et suivantes.

9] W. Dorner, P. Dhmont et D. Chavannes. Microbiologie laitière. Payot, Lausanne, 1942, p. 150, 151 et 156.

[10] SHerw Ood. J. Dairy Res., 1939, X, 326.

[11] A. A. Griffith. Proc. Roy Soc., 1920, 221 à 163.

\title{
DOSAGE PHOTOMÉTRIQUE DES LIPIDES DU LAIT - MIS EN DISPERSION AQUEUSE
}

\author{
par \\ ETIENNE GOIFFON \\ Ingénieur I.A.N. \\ HISTORIQUE
}

Les procédés de dosage de la matière grasse du lait sont extrêmement nombreux. Les deux méthodes le plus couramment employées pour doser pratiquement la matière grasse du lait, sont celles de Gerber et de Hoyberg.

Ces techniques que nous n'indiquerons pas ici ont des avantages et des inconvénients.

Une vieille méthode qui semble eomplètement oubliée et qui a eu son succès, utilise le lactoscope de Donné. Cet appareil néphélémétrique, un peu simpliste, mesurait l'opacité du lait supposée proportionnelle à la quantité de globules gras en dispersion.

En dehors même de l'imprécision de la mesure optique, ce procédé suppose, fixe et uniforme la grosseur des globules gras, ce qui est loin d'être la règle.

Le principe de la méthode n'en était pas moins séduisant à cause de sa grande simplicité, et e'est ce qui nous a incité à étudier si l'une des causes d'imprécision de cette méthode pourrait être éliminée.

Si nous pouvions, en effet, assurer une dispersion, réglée et constante des globules gras, nos appareils actuels de photométrie pourraient permettre un dosage préeis des graisses du lait. 


\section{RECHERCHE DE LA POSSIBILITÉ D'UN DOSAGE NÉPHÉLÉMÉTRIQUE}

Nous avons recherché si on ne pourrait pas tirer une méthode de dosage néphélémétrique de la dispersion dans un excès d'eáu de beurre contenu dans une solution acétonique. On sait qu'il se forme ainsi une émulsion plus ou moins opaque selon l'abondance des lipides contenus et la grosseur des grains de cette dispersion.

Il était impossible de songer à reproduire une émulsion mécanique dans l'eau. des graisses préalablement extraites avec une grosseur de grain définie. Nous ne pouvions done partir que d'une solution préalable des graisses dans un solvant, lui-même soluble dans l'eau par le mélange de cette solution lipidique avec un excèsd'eau, on obtient une émulsion qui semble au premier abord homogène.

Il s'agissait de déterminer dans quelles conditions la grosseur de ces grains pourrait être reproduite à coup sûr par une manipulation simple : dans ces conditions un dosage au photomètre en serait. possible.

La grosseur des grains d'une dispersion est infiniment variable non seulement avec le solvant, mais encore avec le contaminant (ainsi qu'on appelle le corps qui constitue le film interfacial qui sépare entre elles les sphérules dispersées), avec l'agitation, avecla température.

On sait, d'autre part que, sans précaution particulière les émulsions tendent à vieillir et les granules à se réunir; l'opacité du mélange varie avec le temps.

Nous avons étudié chacun des facteurs susceptibles de donner des variations de l'opacité pour une même quantité de beurre mis en jeu.

Nous avons cherché dans la littérature si des méthodes analogues: avaient été élaborées sur des bases expérimentales bien définies.

Nous ne l'avons pas trouvé et nous nous sommes aperçus que le problème que nous nous posions, dépassait de beaucoup le but pratique que nous nous étions assigné. Nous avons vite éprouvé qu'un procédé de cet ordre est extrêmement délicat à mettre au point, que de nombreux facteurs entraient en jeu et qu'il fallait d'abord établir une méthode générale de mesure d'un lipide par l'opacité de son émulsion. Ce micro-dosage pourrait recevoir ensuite des applications multiples, tel que le dosage des lipides du sang, et d'autres produits organiques, de certaines graisses oléagineuses isolées.

Nous ne voulons pas dire par là que les valeurs néphélé- 
métriques obtenues par, ce procédé avec un lipide donné puissent être utilisées pour le dosage dans les mêmes conditions d'un autre lipide, car chaque lipide a sa courbe de référence propre.

\section{Proportionnalité dans les mêmes conditions entre les densités optiques et le taux des lipides}

Il était évident qu'il fallait tout d'abord vérifier que notre hypothèse de départ fondamentale, était exacte, et que, en se maintenant dans des conditions empiriques rigoureusement semblables, il existe une proportionnalité entre les densités optiques et les quantités de lipides en jeu.

Il s'agissait d'utiliser une solution de lipides dans un solvant miscible à l'eau, et de rompre par adjonction d'eau, cette solution en l'amenant à un taux de solvant très inférieur à celui nécessaire pour_dissoudre les lipides. On pouvait ainsi obtenir une dispersion dans une phase aqueuse contenant un taux d'acétone très faible.

Cette dispersion provoque-t-elle un trouble proportionnel à la quantité de lipides mise en jeu ? C'est ce qu'il fallait tout d'abord vérifier dans des expériences où le taux des lipides seuls variait, toutes les autres conditions empiriquement choisies, restant tes mêmes.

Aucune donnée expérimentale connue ne nous permettait d'établir d'emblée les conditions, même approximatives, de nos expériences ; nous nous trouvions dans un domaine totalement inexploré, à notre connaissance, et il nous a fallu un certain nombre de tâtonnements pour déterminer, tout au moins l'ordre de grandeur des facteurs à mettre en jeux. Ces essais nous ont montré plusieurs possibilités (dans les proportions des mélanges notamment) parmi lesquelles nous avons choisi des conditions simples, faciles à réaliser dans chaque cas en utilisant le beurre anhydre ou de l'huile en solution acétonique.

\section{Technique expérimentale.}

Dans une série de verres à expérience, nous plaçons des quantités croissantes de solution acétonique de beurre dont nous complétons le volume à $5 \mathrm{~cm}^{3}$ avec de l'acétone, et nous y mélangeons $30 \mathrm{~cm}^{3}$ d'eau. Il se forme un trouble (le taux de l'acétone étant ramené à $14 \%$ ). On le mesure au photomètre au bout d'un temps déterminé. Les densités optiques obtenues s'inscrivent selon une droite par rapport aux quantités de beurre. 
Exemple :

\begin{tabular}{c|c|c|c}
\hline \hline $\begin{array}{c}\text { Solution acétonique } \\
\text { du beurre } 1 \%\left(\mathrm{~cm}^{3}\right)\end{array}$ & Acétone $\left(\mathrm{cm}^{3}\right)$ & Eau $\left(\mathrm{cm}^{3}\right)$ & Densité optique \\
\hline 0,5 & 4,5 & 30 & 30 \\
1 & 4 & 30 & 50 \\
1,5 & 3,5 & 30 & 71 \\
2 & 3 & 30 & 92 \\
2,5 & 2,5 & 30 & 112 \\
\hline \hline
\end{tabular}

Done, toutes conditions étant égales, les variations de la quantité de lipides dispersés, donnent des troubles dont les densités optiques sont en progression régulière et rectiligne.

Ce fait essentiel étant établi, nous avons modifié systématiquement les variables physiques capables d'entrer en jeu, pour étudier leur ínfluence sur le degré de dispersion obtenu. Ainsi, espéronsnous pouvoir choisir à bon escient les conditions optima pour arriver à un dosage pratique, exempt des incertitudes provenant des. variations produites par ces facteurs.

\section{ETUDES DES VARIABLES .}

\section{A. Mode de mélange.}

Il était possible que la rapidité plus ou moins grande de l'affusion d'eau, dans la solution acétonique de lipides provoquât une variation de la dispersion des granules graisseux. Ce point a été étudié tout spécialement.

Dans une série de verres coniques, on place $3 \mathrm{~cm}^{3} 5$ de solution acétonique de beurre à $1 \%$ et $7 \mathrm{~cm}^{3} 5$ d'acétone auxquels on ajoute plus ou moins rapidement $30 \mathrm{~cm}^{3}$ d'eau contenus dans une éprouvette.

Nous obtenons des densités optiques différentes :

Densité optique

Versé d'un coup ..................... $\quad 99$

Versé assez lentement avec agitation .......... 105

Versé lentement avec agitation $\ldots \ldots \ldots \ldots \ldots \ldots \ldots . \quad 150$

On voit donc que le maximum d'opacité est obtenu par une affusion d'eau lente et progressive, mais nous avons observé que la vitesse de cette affusion est difficile à régler et que les variations qui peuvent être involontaires, provoquent des modifications assez importantes de la densité.

Au contraire, avec une affusion rapide, on obtient des valeurs constantes, ne dépendant pas essentiellement d'un tour de main 
spécial, puisque en ralentissant un peu la rapidité de cetté affusion, on n'obtient qu'une augmentation d'opacité peu importante.

Il est de règle, en cas de variations semblables, de choisir les conditions qui amènent le maximum d'opacité.

VERnes par exemple utilise une émulsion aqueuse d'un antigène lipidique dissous dans l'alcool, pour sa réaction de la syphilis. Il prescrit de la faire en versant goutte à goutte, en un temps donné, l'extrait alcoolique dans l'eau, en-l'agitant avec un appareil rotatif réglé à un nombre de tours minutes défini.

Nous àvons préféré choisir délibérément le procédé d'affusion qui donne le trouble le plus faible, mais le plus constant, le plus indépendant des modifications légères du mode opératoire que Vernes ne régularisait qu'au prix d'un appareillage compliqué.

\section{B. Vieillissement et maturation.}

En utilisant comme précédemment la solution acétonique de lipides et l'eau distillée, nous avons constaté que la lecture peut se faire au bout de 10 minutes sans modification de la densité optique pendant 30 minutes et plus.

En attendant plus longtemps, les densités optiques changent très lentement.

Notons, en passant, qu'au bout de 24 heures, si la densité s'est modifiée, l'émulsion est restée parfaitement homogène.

\section{Température.}

Nous avons constaté que les variations de température autour de la température ordinaire, n'avaient pas d'influence sur les densités optiques obtenues. Ce n'est qu'aux températures au-dessus de $30^{\circ}$ et au-dessous de $10^{\circ}$ que nous avons observé des modifications notables.

\section{Emploi d'un contaminant.}

Dans les émulsions vraies un liquide est dispersé dans un autre sous forme de sphérules.

Deux tensions superficielles s'établissent entre les surfaces de ces particules. Si l'on veut qu'une émulsion de ce genre, entre deux liquides non solubles l'un dans l'autre, soit stable, il faut que la surface de contact entre ces deux liquides soit constituée par une substance légèrement soluble dans l'un et l'autre liquide, il se forme ainsi un revêtement, un film de molécules orientées.

On appelle contaminant, la substance qui constitue ce film interfacial et qui abaisse fortement la tension superficielle. Par là même, elle provoque la formation de très fines sphérules et les isole entre elles. 
a) Nous avons pensé à régulariser et stabiliser la formation de notre émulsion, en utilisant au lieu d'eau pure, une solution à $0,5 \%$. d'oléate de soude.

Ce corps, à très faible concentration, a la propriété d'abaisser fortement la tension superficielle de l'eau, la ramenant à 22 dynes, alors que la tension superficielle de l'eau est de 73 dynes.

$\mathrm{Ce}$ corps tensio-actif, dont les particules tendent à s'absorber en couche extrêmement mince sur les parois, revêt les granules en suspension d'une sorte d'enduit isolant qui favorise et maintient. l'émulsion.

Mais cette adjonction s'est avérée inutile.

L'expérience nous a montré qu'avec ou sans oléate de soude, l'adjonction d'un même volume d'eau à un même volume de solution-acétonique de lipides, donnait exactement la même densité optique.

Malgré tout, le pouyoir d'étalement des oléates alcalins, est tellement grand que nous ne pouvons affirmer qu'une trace de ces: savons n'existait pas dans le beurre employé et, au cas ou il s'agirait du dosage d'une huile ou d'un beurre rigoureusement pur, nous préconiserons à tout hasard, l'adjonction de $0 \mathrm{gr}$. I d'oléate de soude pár litresde la solution aqueuse servant à la précipitation ;

b) On sait, d'autre part, que les émulsions sont stabilisées par la présence d'un colloïde visqueux tel que les gommes.

Nous avons essayé d'utiliser également une solution aqueuse d'alginate de soude à 1 gramme par litré ; les résultats n'ont pas été différents de ceux obtenus avec de l'eau pure.

Il semble, en réalité que le contaminant nécessaire soit constitué par l'acétone elle-même, qui possède la qualité requise d'être soluble à la fois dans l'eau et dans l'huile et d'abaisser la tension superficielle de ses solutions aqueuses.

\section{Influence de la proportion entre l'acétone et l'eau.}

Si nous reprenons notre première expérience par laquelle nous. avions constaté l'existence d'une proportionnalité entre les quantités de lipides dispersés et la densité optique de cette dispersion, en modifiant simplement les proportions respectives d'acétone et d'eau $(42,5 \%$ d'acétone au lieu de $14 \%$, nous obtenons des opacitésdifférentes pour un même poids de lipides.

Les proportions respectives d'eau et d'acétone ayant été fortement modifiées, lorientation des courbes est changée ; les densités optiques restent cohérentes pour chaque système, mais ce ne sont pas les mêmes pour un même poids de lipides. 
Exemple :

\begin{tabular}{c|c|c|c}
\hline $\begin{array}{c}\text { Solution acétonique } \\
\text { de beurre à } 1 \% \\
\left(\mathrm{~cm}^{3}\right)\end{array}$ & Acétone $\left(\mathrm{cm}^{3}\right)$ & Eau $\left(\mathrm{cm}^{3}\right)$ & Densité optique \\
\cline { 2 - 3 } 1,5 & 13,5 & 15 & 33 \\
2 & 13 & 15 & 48 \\
2,5 & 12,5 & 15 & 60 \\
3 & 12 & 15 & 75 \\
3,5 & 11,5 & 15 & 86,5 \\
4 & 11 & 15 & 102 \\
\hline
\end{tabular}

Nous voyons done dans cette première expérience que la quantité d'acétone dans laquelle est dissoute le beurre ou plus exactement la concentration finale d'acétone du mélange aqueux, détermine la grosseur micellaire de l'émulsion. Celle-ci reste la même avec des variations de la concentration des lipides.

\section{Progression des densités optiques suivant les taux d'acétone de l'émulsion}

Il était intéressant d'étudier quelle allure prend une courbe traduisant les densités optiques d'une émulsion contenant sous un même volume une quantité uniforme de lipides, mais avec des proportions variées d'acétone et d'eau.

Expérience (fig. 1) ?

\begin{tabular}{c|c|c|c|c}
\hline \hline $\begin{array}{c}\text { Solution acéto- } \\
\text { nique de beurre } \\
\text { à } 1 \%\left(\mathrm{~cm}^{3}\right)\end{array}$ & $\begin{array}{c}\text { Acétone } \\
\left(\mathrm{cm}^{3}\right)\end{array}$ & Eau $\left(\mathrm{cm}^{3}\right)$ & $\begin{array}{c}\text { Concentration } \\
\text { finale d'acét. }\end{array}$ & $\begin{array}{c}\text { Densité optique } \\
\text { Bonnet-Maur }\end{array}$ \\
\hline 5,5 & 7 & 20 & 34,4 & 40 \\
3,5 & 8 & 19 & 37,7 & 30 \\
3,5 & 9 & 18 & 40 & 27 \\
3,5 & 10 & 17 & 44,2 & 26 \\
3,5 & 11 & 18 & 47,5 & 25 \\
3,5 & 12 & 15 & 50,8 & 26 \\
3,5 & 13 & 14 & 54 & 28 \\
3,5 & 14 & 13 & 57,3 & 30 \\
3,5 & 15 & 12 & 60,6 & 39 \\
\hline \hline
\end{tabular}

Nous voyons par cette courbe que la densité optique diminue au fur et à mesure que s'accroît la quantité d'acétone, arrive à un 


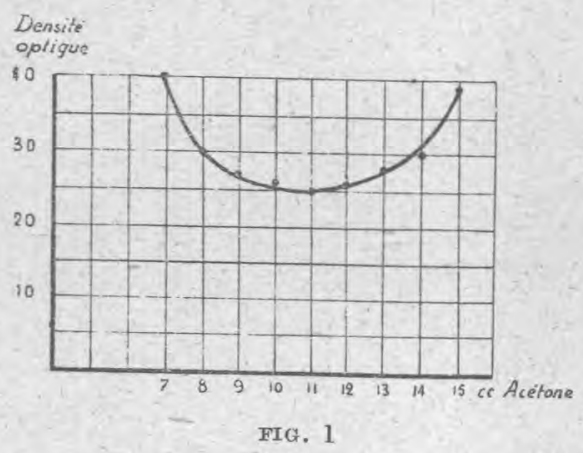

minimum aux environs de $50 \%$ et se relève quand la proportion d'a. cétone l'emporte sur celle de l'eau.

Variations d'acétone pour quantités différentes de lipides

Ce même phénomène peut être mis en évidence en déterminant des segments de courbes tracées avec quatre mêmes quantités de beurre, avec le même volume de dilution finale mais avec des proportions variables d'acétone.

\begin{tabular}{c|c|c|c}
\hline \hline $\begin{array}{c}\text { Solution acétonique } \\
\text { du beurre à } 1 \% \\
\left(\mathrm{em}^{3}\right)\end{array}$ & Acétone $\left(\mathrm{cm}^{3}\right)$ & Eau $\left(\mathrm{cm}^{3}\right)$ & Densité optique \\
\cline { 2 - 3 } 1 & & & \\
\hline 1 & 7 & 22 & 14 \\
1 & 9 & 20 & 13 \\
2 & 11 & 18 & 14 \\
2 & 6 & 22 & 39 \\
2 & 8 & 20 & 36 \\
3 & 10 & 18 & 35 \\
3 & 5 & 22 & 80 \\
3 & 7 & 20 & 69 \\
4 & 9 & 18 & 559 \\
4 & 4 & 22 & 154 \\
4 & 6 & 20 & 117 \\
4,5 & 8 & 18 & 105 \\
4,5 & 3,5 & 22 & 208 \\
4,5 & 5,5 & 20 & 145 \\
& 7,5 & 18 & 133 \\
\hline \hline
\end{tabular}

Ce tableau nous montre que les variations dues au volume d'acétone sont presque insensibles avec de faibles concentrations de beurre, mais qu'elles sont considérables lorsque la proportion de beurre dispersé est plus grande.

Si l'on admet qu'une très grande dispersion avec micelles très petites se rapproche de l'état colloïdal, transparent, qu'une dispersion en molécules grossières fournit un trouble moins grand que des particules plus divisées, on arrive à concevoir d'après notre 
tableau que cette division est à son maximum avec $50 \%$ d'acétone.

On peut conclure également d'après le tableau que plus la concentration des lipides en solution acétonique est grande et plus la grosseur des micelles s'éloigne de l'état colloïdal.

\section{E. Variations du $p \mathrm{H}$.}

Toutes les expériences précédentes ont été pratiquées avec de. l'eau distillée proche de la neutralité.

Quand nous avons exploré les variations de la réaction du mélange, nous avons vite constaté que l'adjonction d'un peu. d'acide ou d'alcalin changeait considérablement le degré de dispersion du mélange et nous a incité à faire une étude systématique des. variations du $p \mathrm{H}$.

Pour cela nous avons employé soit de la soude ou de l'HCl pur, soit des sels acides et alcalins, soit des mélanges tampons - nous avions auparavant vérifié que l'adjonction à notre liquide de précipitation, d'une petite quantité d'électrolyte, ne change pas son pouvoir dispersant.

Nous avons observé :

1. Que pour unè même quantité d'acétone contenant la même quantité de lipides, dans laquelle on projette une solution aqueuse à $p H$ variable, l'opacité la moins grande répondant à l'état le plus colloïdal se trouve aux environs de la neutralité.

2. Que plus l'acidité s'élève, plus l'opacité du trouble augmente et qu'il en est exactement de même si c'est l'alcalinité qui s'accrôit.

La courbe suivante résume les données que nous avons observées.

Ce fait important nous incitait à choisir quelles zones il serait préférable de choisir en vue d'un dosage pratique. Il est évident, d'après la lecture de cette courbe, que trois atfitudes étaient possibles.

10 On pourrait se placer à $p H ~ 7$ en utilisant un tampon correspondant à la concentration de ces

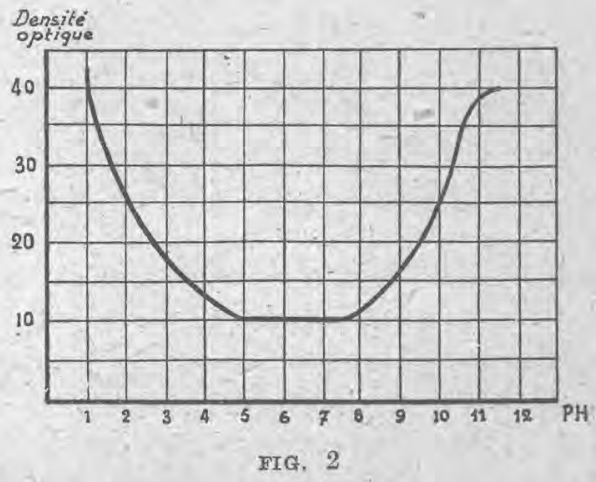
ions $H$, ce qui est indispensable pour éviter les fluctuations provenant de l'acidité fortuite de l'eau distillée ou des lipides à mesurer. 
Les moindres variations vers l'acidité entraîneraient d'après les formes mêmes de notre courbe, des variations extrêmement rapides qui fausseraient a priori, tous dosages. Ce choix de la neutralité nous ramènerait aux conditions dans lesquelles nous nous trouvions dans les essais expérimentaux tels que nous les avions écrits et qui donnaient entière satisfaction.

Cependant, étant donné le but pratique que nous avons en vue, l'utilisation d'un tampon à $p$ H 7 , est relativement peu pratique, et l'expérience montre que la moindre complication diminue singulièrement les adeptes de cette méthode.

Nous a vions donc à choisir entre la zone acide et la zone alcaline;

$2^{\circ}$ Il était à prévoir, d'après notre courbe, qu'il était indispensable d'évitẹr les zones intermédiaires de l'échelle des $p H$ pour nous placer aux concentrations maxima, c'est-à-dire, entre $p \mathrm{H}: 1$ et 0 . Nous avons employé une solution d' $\mathrm{HCl}$ à 5 pour 1.000 et nous avons repris toutes les expériences précédentes.

Notre premier soin a été de savoir avee quelle rapidité la densité optique maxima se stabilisait et si de nombreuses expériences faites dans ces conditions apparemment semblables donnaient les mêmes résultats.

Nous nous sommes arrêté à ce premier stade car nous assistions à une ascension continue des densités optiques qui se poursuivaient insensiblement pendant des heures sans qu'un plateau constant puisse être atteint au bout d'un même temps. Sans que nous ayons pu en déceler les raisons, une même expérience répétée à plusieurs reprises, subissait de légères variations au bout d'un même temps.

$3^{\circ}$ Nous avons done reporté notre attention vers la zone alcaline. Nous avons trouvé dans le $\mathrm{CO}^{3} \mathrm{Na}^{2}$ une substance capable d'assurer un $p \mathrm{H}$ constant du mélange qui nous a donné entière satisfaction. Mais nous avons dû reprendre dans ces nouvelles conditions toutes les expéríences que nous avions faites avee de l'eau distillée pure.

Nous avons employé le $\mathrm{CO}^{3} \mathrm{Na}^{2}$ dans le but de rendre la réaction plus sensible, mais dans le cas du lait il peut être plus pratique et plus intéressant d'utiliser la dispersion à $p H$ près de la neutralité en employant une quantité un peu plus grande de lipides comme nous l'avons fait dans la première partie de ce travail. Il suffira de s'en tenir au choix qui a été fait $d u \quad p H$ de ce travail et établir les courbes de références sur une solution titrée de beurre au $p \mathrm{H}$ choisi et d'opérer dans les mêmes conditions pour le beurre extrait des laits à examiner. 


\section{Mode de mélange.}

Le mode de mélange dans ces conditions a eu un effet complètement opposé à celui qu'il avait avec de l'eau distillée ; nous avions trouvé, en effet, qu'un mélange lent fournissait des densités optiques plus grandes qu'un mélange rapide. Avec le $\mathrm{CO}^{3} \mathrm{Na}^{2}$ au contraire la dénsité optique la plus intense a été provoquée par l'affusion rapide de l'eau carbonatée, fait dont nous n'avons pu trouver les raisons.

Comme la vitesse du mélange pouvait, au point de vue pratique, être une cause d'erreur, il eut fallu pour les mélanges lents déterminer sans doute à la fois la vitesse d'affusion et la vitesse d'agita. tion, ce qui a été réalisé pour l'obtention des suspensions standard de l'antigène de Vernes.

Il était plus simple de choisir le mélange brusque dont les modalités prêtent à moins de variations. Cependant pour nous assurer qu'un tour de main spécial n'était pas requis pour obtenir la même grosseur de grains, nous avons pratiqué le mélange en projetant l'eau brutalement dans un grand verre à pied dont rien ne pouvait s'échapper et, d'autre part, l'eau a été versée rapidement, mais avec précaution, dans un verre de capacité restreinte comme aurait pu le faire un expérimentateur timoré. Nous encadrons ainsi toutes les modalités de la « brusquerie » exigée pour le mélange. Nous avons constaté que les deux valeurs obtenues dans ces conditions étaient égales.

\section{Progression des $D$. optiques suivant la proportion d'acétone et d'eau.}

Dans nos premières expériences à $p H \quad 7$, nous avions constaté que, en faisant varier les quantités d'acétone et d'eau, pour un même volume final, nous obtenions une courbe d'allure parabolique dont le minimum s'obtenait avec les proportions égales d'acétone et d'eau.

En reprenant cette expérience avec le $\mathrm{CO}^{3} \mathrm{Na}^{2}$ à $p \mathrm{H}: 11,3$, le phénomène est complètement différent : on obtient les densités optiques les plus fortes avec $5 \mathrm{~cm}^{3}$ d'acétone pour $30 \mathrm{~cm}^{3}$ d'eau carbonatée. Si on augmente les quantités d'acétone en diminuant proportionnellement celles de l'eau on obtient un abaissement progressif et constant de la D. optique.

Là aussi nous n'expliquerons pas cette différence dans le comportement des mélanges acétoniques neutres et alcalins dont les. différentes charges électriques sont évidemment responsables. 


\section{Vieillissement et température.}

La densité optique du mélange augmente pendant 40 minutes et reste stable pendant quelques heures.

Les variations de température de l'eau carbonatée employée n'ont pas d'influence entre les $15^{\circ}$ et $28^{\circ}$ auxquels nous les avons essayé.$$
*^{*} *
$$ \\ Technique d'un dosage des graisses}

L'ensemble de ces recherches nous a permis de déterminer les conditions qui nous semblaient optima pour le dosage néphélométrique du dosage des graisses, Les conditions sont celles qui sont les moins sujettes à des variations accidentelles dans la manipulation.

Un échantillon à doser de l'ordre de grandeur de 10 milligrammes qui sont mis en solution dans $10 \mathrm{~cm}^{3}$ d'acétone dans un bécher ou un grand verre à pied d'au moins $100 \mathrm{~cm}^{3}$. On projette dans cette solution acétonique, $30 \mathrm{~cm}^{3}$ d'eau distillée contenant 30 grammes de $\mathrm{CO}^{3} \mathrm{Na}^{2}$ cristallisé par litre. Une partie de ce mélange bien agité est placé dans un tube à essai et bouché pour éviter l'évaporation de l'acétone. Au bout de 30 minutes, on mesure la densité optima au photomètre. On aura auparavant tracé une courbe avec des quan-

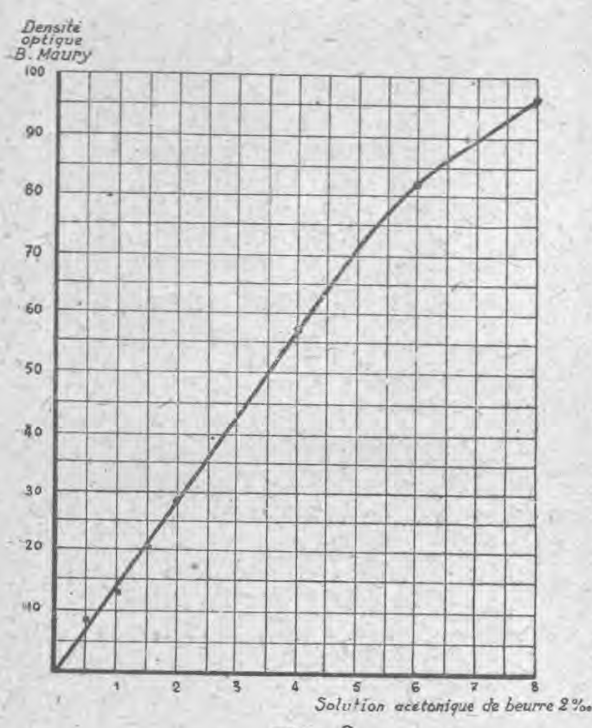

FIG. 3 tités croissantes de graisses trai tées dans les mêmes conditions.

En pratique, on utilise une solution acétonique de beurre deshydraté ou d'huile (selon la graisse) que l'on se propose de doser à $2 \%$; on en prélève 2,4 , $6,8,10 \mathrm{~cm}^{3}$; on complète à 10 $\mathrm{cm}^{3}$ avec de l'acétone chaque prise d'essai et on pratique la dispersion cómme précédemment avec $30 \mathrm{~cm}^{3}$ d'eau carbonatée, et on détermine la densité optique de chacun des mélanges, au bout de 30 minutes.

Cette courbe de référence permet de déterminer les relátions de la densité optima obtenue avec le poids de graisse en jeu.

Nous ferons remarquer que les intensités de la densité optique avec la même quantité de graisse sont incomparablement plus faibles en milieu neutre qu'en milieu alcalin et que, par conséquent, 
le choix que nous faisons désormais du dosage en milieu alcalin comporte une prise d'essai plus petite que celle qui nous a servi dans nos expériences antérieures en milieu neutre. Ces courbes que nous avons dans les deux cas ne peuvent done être comparées au point de vue quantitatif.

\section{APPLICATION AU LAIT}

Pour le lait, l'extraction du beurre peut être faite avec la technique classique d'Adam-Meillière.

Si l'on attribue au lait une teneur de 30 grammes pour 1.000 de beurre, $1 \mathrm{~cm}^{3}$ en contiendrait done 30 milligrammes. Il suffira, soit de faire une extraction sur un quart de centimètre cube de lait, en diminuant proportionnellement la quantité des lipides extractifs, soit d'employer une plus grande quantité de lait. Mais après l'obtention du résidu de beurre obtenu après évaporation de l'éther, il suffira de dissoudre ce résidu dans un volume déterminé d'acétone et d'en prélever pour le dosage une part aliquote, contenant 3 à 6 milligrammes de matière grasse diluée par de l'acétone à un volume de de $10 \mathrm{~cm}^{3}$.

\section{Technique sur un lait normal}

Mettre $1 \mathrm{~cm}^{3}$ de lait bien homogénéisé dạns ampoule à décantation Ajouter $4 \mathrm{~cm}^{3}$ d'alcool sodé $+5 \mathrm{~cm}^{3}$ d'éther. Agiter, soutirer la partie aqueuse, mettre la partie éthérée dans une capsule et faire évaporer. Laver la capsule avec $10 \mathrm{~cm}^{3}$ d'acétone. Prendre $\left(2 \mathrm{~cm}^{3}\right.$ correspondant à $0 \mathrm{~cm}^{3} 2$ de lait) et amener avec de l'acétone à un volume de $10 \mathrm{~cm}^{3}$. On projette $30 \mathrm{~cm}^{3}$ d'eau carbonatée. Il se forme un trouble qu'on mesure au bout de 30 minutes.

\section{Exemple :}

Soit une D. optique B. M. (écran 15) $=46$ correspondant à 65 milligrammes de matières grasses, nous aurons $65 \times 5 \times 1.000=$ 32 gr. 5 de matières grasses par litre de lait.

Ce procédé n'ẻvite, en somme, que la pesée puisqu'elle suppose une extraction préalable du beurre avec évaporation du liquide extractif.

II peut rendre eependant des services au cas où la quantité de lait (lait de femme, de petits animaux) ne permettrait pas d'en disposer de quantités suffisantes pour une pesée rigoureuse.

Nous avons essayé d'adapter notre procédé à des eonditions plus pratiques et plus rapides pour l'industrie laitière.

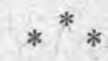




\section{Essai d'application à l'industrie laitière}

Etant donné que nous pouvons doser néphélémétriquement les lipides isolés en solution acétonique, tout le problème consistait à trouver une méthode pratique d'extraction du beurre du lait sans être obligé de passer par l'alcool éther et l'évaporation préalable comme dans la méthode Adam-Meillère. Nous avons essayé deux procédés :

\section{Extraction directe par l'acétone.}

Nous avons essayé de projeter $1 \mathrm{~cm}^{3}$ de lait dans $19 \mathrm{~cm}^{3}$ d'acétone mais l'extraction n'était pas parfaite, étant donné la grosseur variable des caillots formés pendant cette opération. On a d'excellents résultats en versant goutte à goutte $1 \mathrm{~cm}^{3}$ de lait dans $10 \mathrm{~cm}^{3}$ d'acétone continuellement agitée dans un Erlenmeyer.

On peut également, pour multiplier les surfaces de lait accessibles à l'acétone, mouiller avec $1 \mathrm{~cm}^{3}$ de lait des billes de verre bien sèches placées dans le fond d'un Erlenmeyer, - également sec, - et en quantité suffisante pour que le lait soit entièrement réparti à leur surface. On projette ensuite $19 \mathrm{~cm}^{3}$ d'acétone, et on agite soigneusement le tout, en laissant au contact pendant $5 \mathrm{mi}$ nutes et en ayant soin de boucher le flacon afin qu'il n'y ait pas. d'évaporation. On décante ensuite une partie du liquide acétonique qui est presque limpide, on le filtre rapidement - on prélève de ce filtrat $4 \mathrm{~cm}^{3}$ (correspondant à un cinquième de centimètre cube de lait et par conséquent pour un lait normal 6 à 8 milligrammes de beurre) qu'on place dans un verre à pied ; on ajoute $6 \mathrm{~cm}^{3} \mathrm{~d}^{\prime}$ acétone pour que le volume final d'acétone soit égal à $10 \mathrm{~cm}^{3}$, puis on projette $30 \mathrm{~cm}^{3}$ d'eau carbonatée. Nous obtenons un trouble que nous lisons au bout de 30 minutes au photomètre.

Les dosages que nous avons faits dans ces conditions comparés aux dosages pondéraux par la méthode Adam-Meillère sur les. mêmes échantillons-de lait, nous ont donné des chiffres un peu moins précis, mais qui cependant pourraient fournir une indication pratique suffisante dans certains cas.

Nous avons constaté que de petites quantité d'éleetrolytes et de lactose telles que celles qui pouvaient être introduites avec le lait ne changeaient pas le degré de dispersion des lipides. Les erreurs sont vraisemblablement attribuables à l'incertitude de l'extraction lipidique directe par l'acétone.

On sait qu'il est possible de remplacer les photomètres plus ou moins dispendieux par le colorimètre de Duboseq én lumière monochromatique et en remplaçant l'un des godets par un écran légèrement opaque dont il est facile d'étalonner la valeur néphélémétrique. 


\section{Dispersion directe du mélange éthéro-alcoolique.}

Nous nous sommes demandé si nous ne pouvions pas assurer avee régularité la dispersion des lipides en employant comme prise d'essai, le mélange éthéro-alcoolique, utilisé dans la méthode Adam-Meillère.

Nous avons remplacé l'alcool ammoniacal par l'acleool sodé utilisé par Grigaut pour l'extraction du cholestérol du sérum sanguin ( $1 \mathrm{~cm}^{3}$ de lait $+4 \mathrm{~cm}^{3}$ alcool sodé à $60^{\circ}+5 \mathrm{~cm}^{3}$ éther $)$.

La phase aqueuse était soigneusement soutirée, le liquide alcool-éther était versé dans une éprouvette de $10 \mathrm{~cm}^{3}$ et amené à l'acétone au volume de $10 \mathrm{~cm}^{3}$. On en prend $2 \mathrm{~cm}^{3}$ que l'on place dans un verre à pied avec $8 \mathrm{~cm}^{3}$ d'acétone.

On ajoute d'un coup $30 \mathrm{~cm}^{3}$ d'eau carbonatée à $30 \%$, on lit la densité optique au bout de 30 minutes.

Nous avons obtenu des résultats cohérents dans ces conditions. Une courbe de référence doit être faite avec des solutions de beurre fondu dont chaque prélèvement doit comporter exactement la même proportion d'alcool et d'éther que dans notre mélange d'extraction éthéro-alcoolique.

Dans ces conditions, l'éther est solubilisé dans l'eau suffisamment pour ne fournir aucun, trouble supplémentaire. En réalité un excès d'éther diminue le trouble jusqu'à le supprimer. C'est cette difficulté de manipulation d'une substance aussi volatile que l'éther dont la concentration peut changer suivant la température du laboratoire, le temps des manipulations, qui nous a fait aban. donner, provisoirement, ce procédé.

II serait cependant possible d'éviter ces inconvénients et d'en tirer une mẻthode pratique qui serait évidemment un procédé de choix à cause de sa rapidité pour une mesure des lipides du lait.

\section{RÉSULTATS}

PREMì̀re Méthode.

Exxtraction éthéro-alcoolique et dessication

\begin{tabular}{|c|c|c|}
\hline Lait & $\begin{array}{c}\text { Pesée } \\
\text { (Grammes de matière } \\
\text { grasse par litre) }\end{array}$ & $\begin{array}{c}\text { Dispersion aqueuse } \\
\text { (Grammes de matière } \\
\text { grasse par litre) }\end{array}$ \\
\hline $1 \ldots \ldots \ldots \ldots \ldots$ & 32,5 & 32,1 \\
\hline $2 \ldots \ldots \ldots \ldots \ldots \ldots$ & 32,5 & 32,1 \\
\hline $3 \ldots \ldots \ldots \ldots \ldots \ldots$ & 28,2 & 29,8 \\
\hline
\end{tabular}


DEUUIK̀ME MÉTHODE.

Extraction acétonique

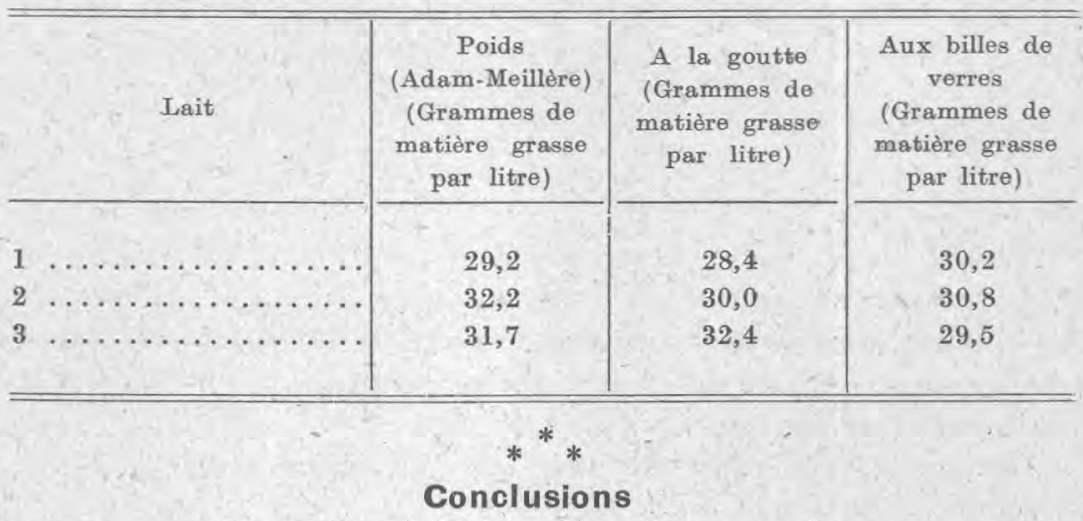

Nous avons montré la possibilité d'un dosage turbidométrique des lipides en solution acétonique dispersée dans l'eau. Il existe, en effet, un rapport rigoureux entre les densités optiques d'une émulsion faite dans les mêmes conditions et les quantités de lipides dispersées.

Nous avons établi le rôle des facteurs possibles de variation : mode de mélange, emploi d'un contaminant, vieillissement, température. La proportion entre l'acétone et l'eau a une importance considérable que nous avons définie et mesurée. L'influence du $p \mathrm{H}$ a été déterminée.

Nous donnons une technique précise pour établir une courbe de référence en vue d'un dosage.

Cette méthode rapide permet, entre autre, un dosage des graisses du lait sur un très petit volume. Nous en avons décrit la technique.

Nous avons donné, en outre, des simplifications fournissant. des valeurs approchées, utilisables à cause de leur rapidité, dans. l'industrie du lait.

$$
\text { *** }
$$

Quelle est la place possible de la méthode proposée parmi les. méthodes classiques de dosage des laits?

Nous sommes loin de proposer notre méthode eomme devant être substituée aux méthodes-classiques par extraction volumétrique telle que Gerber, soit même par la pesée (Adam-Meillière). L'emploi d'un photomètre notamment, limite singulièrement l'emploi pratique du procédé.

Cependant l'exactitude relative des résultats, la simplieité des. manipulations, nous font penser que notre travail peut être à l'origine d'une méthode vraiment pratique et précise elle le serait 
si deux conditions étaient réalisées, l'une, que nous n'avons pas pu maîtriser, est l'extraction rapide et complète des graisses du lait par l'acétone, ou un autre solvant ayant les mêmes qualités de solution dans l'eau.

La deuxième condition serait de posséder un appareil de mesure des troubles aussi simple que le lactoscope de Donné mais plus précis, ee qui n'est peut être pas irréalisable, dans l'avenir. C'est à ce moment, croyons-nous, que nos recherches pourraient avoir un maximum d'efficacité.

\section{SUR L'ÉTAT DE L'ANHYDRIDE CARBONIQUE DU LAIT} par

\section{Arnaldo FOSCHINI}

Dans une note précédente (1) regardant les variations du contenu en anhydride carbonique du lait pendant les transports et les procédés thermiques d'assainissement et de conservation, j'ai eu loccasion de faire allusion à l'importance chimique et biologique attribuée à la présence de ce gaz dans le lait, non seulement de ma part, mais surtout de la part de savants parmi lesquels Stassa no (2).

Il n'est naturellement pas facile de spécifier les raisons qui soutiennent une semblable affirmation ; toutefois, il est permis de présumer que l'anhydride carbonique est présent dans le lait, non fortuitement (en effet le nourrisson ner perd rien avec la tétée), et qu'il doit avoir une influence notable sur les équilibres d'un milieu aussi complexe. Qu'il suffise de penser à cet égard que l'anhydride carbonique, tout en se comportant apparemment comme un acide faible, est en effet un acide d'une force moyenne. THIEL et ses Collaborateurs ont démontré que dans une solution $0,00812 \mathrm{~mol}$. g. de $\mathrm{CO}^{2}$ à $4^{\circ} \mathrm{C}$., seulement $0,67 \%$ est hydraté, mais que cette partie a un degré de dissociation de $91 \%$.

Cela porte à attribuer à l'acide carbonique une constante de dissociation de $5,0 \times 10^{4}$ au lieu de la note constante apparente de $3,0 \times 10-7$.

Les incertitudes qui entourent encore la connaissance de l'intime constitution du lait, empêchent de se faire une idée exacte de l'état dans lequel l'anhydride carbonique vient à se trouver et par conséquent des effets qu'il peut y produire.

On sait que longtemps on a retenu que le $\mathrm{CO}^{2}$ du lait fut tout entier à l'état libre. VAN SLYKE et BAKER (3) pourtant, étudiant

(1) A. Foschini et M. Talenti, Ann. Ig., 1942, LII, 69 .

(2) Le Lait, 1933, XIII, 85.

(3) Journal Biol. Chem., 1919, XL, 335. 